\title{
A BENDIXSON-DULAC THEOREM FOR SOME PIECEWISE SYSTEMS
}

\author{
LEONARDO P. C. DA CRUZ AND JOAN TORREGROSA
}

\begin{abstract}
The Bendixson-Dulac Theorem provides a criterion to find upper bounds for the number of limit cycles in analytic differential systems. We extend this classical result to some classes of piecewise differential systems. We apply it to three different Liénard piecewise differential systems $\ddot{x}+f^{ \pm}(x) \dot{x}+x=0$. The first is linear, the second is rational and the last corresponds to a particular extension of the cubic van der Pol oscillator. In all cases, the systems present regions in the parameter space with no limit cycles and others having at most one.
\end{abstract}

\section{INTRODUCTION}

The study of the number of isolated periodic orbits, the so called limit cycles, is a very relevant problem in the qualitative theory of differential equations. This question, known as the 16th Hilbert problem, was proposed by Hilbert in a list of 23 problems in the International Congress of Mathematics in 1900. In his opinion the study of them would motivate advances in mathematics during the 20th century. The 16th Hilbert problem is one of the few that remain open, see [17]. Now, this problem consists in two different questions: The first asks about the relative positions of the branches of real algebraic curves of some degree. The second asks about the existence of an upper bound for the number of limit cycles in planar polynomial vector fields of degree $n$ besides their relative position. This maximum number depending only on $n$ is usually known as the Hilbert number $H(n)$. There are a lot of published works in relation with this problem. As linear systems have no limit cycles, the most simple case to start with is the quadratic family. But even in this case the problem remains open. The highest value, found in 1980, for this number in quadratic vector fields, which is conjectured that will be the maximum, is $H(2) \geq 4$, see [25]. Recently, this problem has been extended also to piecewise differential equations. In this field, denoting it by $H_{p}(n)$ when the discontinuity line is a straight line, the best result for the simplest case, found in 2012 , says that $H_{p}(1) \geq 3$, see $[1,16,21]$.

There are few specific families that this problem is totally solved. One of the most useful tools to address this question has been the well-known Bendixson-Dulac Theory. It comes to the end of the 19th century, and it appears in most textbooks on differential equations; see for example [8]. Analogously to Lyapunov functions, the main difficulty in this theory is how the so called Dulac function is obtained for the region where the result applies. The recent work [13] presents an interesting extension of the BendixsonDulac theory to regions with holes, where the problem of knowing these Dulac functions is translated to control the sign of a polynomial of one variable in a suitable domain. These ideas can be also found in [4].

With the same aim that Hilbert, in 1998, Smale proposed a list of mathematical problems for the 21st century, see [26]. He also includes a modern version of 16th Hilbert problem, saying that the computation of the Hilbert number can be notably difficult. So, he suggests to prove it for some special class of differential equations where the finiteness,

2010 Mathematics Subject Classification. Primary 34C07, 34C23, 37C27.

Key words and phrases. Piecewise vector field, Uniqueness of limit cycle, Bendixson-Dulac theory. 
even the upper bounds, could be simpler. This is the case of the second order polynomial Liénard equation, $\ddot{x}+f(x) \dot{x}+x=0$, that we can write as the planar system

$$
\left\{\begin{array}{l}
\dot{x}=y-F(x) \\
\dot{y}=-x
\end{array}\right.
$$

Here $F(x)=\int_{0}^{x} f(s) d s$ is also a real polynomial. These systems are so relevant because many areas, as biology, chemistry, engineering,..., use it for modeling real situations. In particular, they were intensely studied to model oscillating circuits. Some elegant results concerning the existence of limit cycles for Lienard's equation were obtained by Filippov in [9]. Bendixson-Dulac theory is a classical approach to provide non existence periodic orbit results. But usually, the most common way to prove uniqueness for these type of equations is the study of the stability of such periodic orbits, via Green's Theorem. Some results providing conditions for $F$ assuring that the limit cycle, when it exists, is unique can be found in [28]. Only for $n=3$ and $n=4$ the 16th Hilbert problem for (1) is solved, see $[18,20]$. But in general this is still an open problem.

The study of piecewise differential systems has been extremely effective in helping to understand the behavior of many important physical phenomena such as fluid flows, elastic deformation, nonlinear optical and biological systems, see [7]. One of the important applications are the impact oscillators that also are to describe by second order differential equations. The aim of this paper is to present an extension of the classical results of Bendixon and Dulac to some classes of piecewise Liénard systems.

Let 0 be a regular value of a function $h: \mathbb{R}^{2} \rightarrow \mathbb{R}$. We denote the discontinuity line by $\Sigma=h^{-1}(0)$ and the two regions by $\Sigma^{ \pm}=\{ \pm h(x, y)>0\}$. Thus, we consider the $\Sigma$-piecewise differential system

$$
Z^{ \pm}=\left\{\begin{array}{l}
\dot{x}=X^{ \pm}(x, y), \\
\dot{y}=Y^{ \pm}(x, y),
\end{array} \quad \text { if }(x, y) \in \Sigma^{ \pm},\right.
$$

where $X^{ \pm}$and $Y^{ \pm}$are $\mathcal{C}^{1}$ functions in $\Sigma^{ \pm}$. Moreover, it is defined on $\Sigma$ following Filippov's convention, see [10] and Figure 1. That is, the points on $\Sigma$ where both vectors fields simultaneously point outward or inward from $\Sigma$ define the escaping or sliding region. The complement in $\Sigma$ defines the crossing region. In fact, the boundary of the escaping/sliding regions is defined by the tangential points of $Z^{ \pm}$in $\Sigma$.

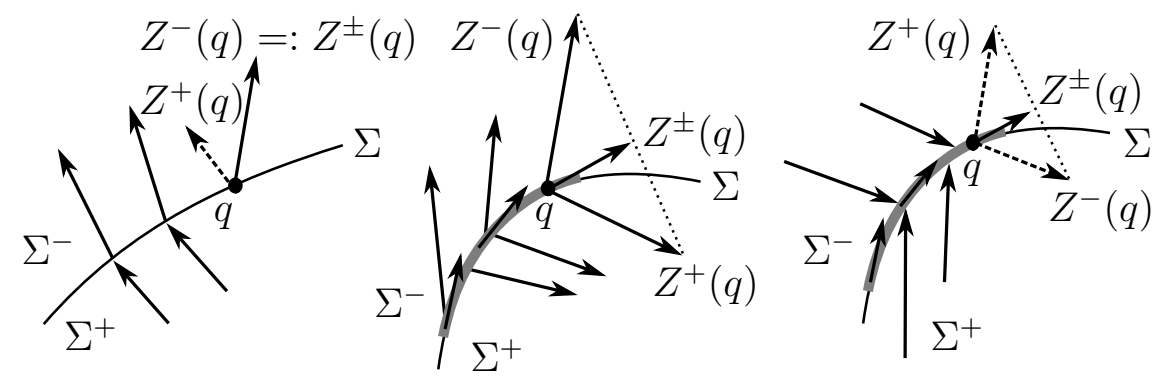

Figure 1. Definition of the vector field on $\Sigma$ following Filippov's convention in the crossing, escaping, and sliding regions.

In this paper, we are interested only in the crossing limit cycles. They are isolated periodic orbit intersecting the discontinuity line on the crossing region. Moreover, we introduce a class of piecewise differential vector fields. They have the next special property on the discontinuity line $\Sigma$. 
Definition 1.1. We say that (2) is a $\Sigma_{P}$-continuous piecewise differential system if it satisfies $Z^{+}(q) \cdot \nabla h(q)=Z^{-}(q) \cdot \nabla h(q)$ for $q \in \Sigma$. Where $\nabla h=(\partial h / \partial x, \partial h / \partial y)$. See Figure 2.

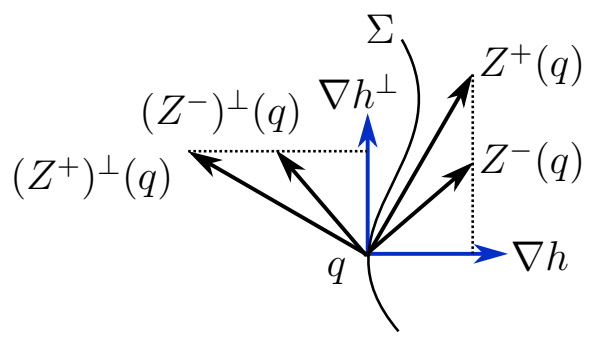

Figure 2. The system (2), and its orthogonal. Both are $\Sigma_{P^{-} \text {continuous }}$ piecewise system.

In Section 2 we will use such property but with the respective orthogonal vectors fields, $\left(Z^{+}(q) \cdot \nabla h(q)\right)^{\perp}=\left(Z^{-}(q) \cdot \nabla h(q)\right)^{\perp}$. Because, sometimes is simpler to check the $\Sigma_{P^{-}}$ continuous property with the tangent vector of the discontinuity line. See also Figure 2.

The following result provide conditions to bound the number of limit cycles for some piecewise systems (2). It will be a generalization of the classical Bendixon-Dulac Theorem to piecewise differential systems.

We say, as in [13], that $S \subset \mathbb{R}^{2}$ is an $\ell$-connected region if its fundamental group, $\pi_{1}(S)$, is $\mathbb{Z} * \cdots *(\ell) * \mathbb{Z}$, or in other words, if $S$ has $\ell$ holes.

Theorem 1.2. Let $S \subset \mathbb{R}^{2}$ be an $\ell$-connected region with a boundary defined by a finite number of smooth pieces and $S^{ \pm}=S \cap \Sigma^{ \pm}$. Consider system (2) defined in the region $S$, $B^{ \pm}: S^{ \pm} \rightarrow \mathbb{R}$ a $\mathcal{C}^{1}$-piecewise function, and $Z_{B}^{ \pm}$the $\Sigma_{P}$-continuous piecewise vector field

$$
Z_{B}^{ \pm}=\left(B^{ \pm}(x, y) X^{ \pm}(x, y), B^{ \pm}(x, y) Y^{ \pm}(x, y)\right) \text { if }(x, y) \in \Sigma^{ \pm} .
$$

If $\operatorname{div}\left(Z_{B}^{+}\right) \cdot \operatorname{div}\left(Z_{B}^{-}\right) \geq 0$ and $\left(\operatorname{div}\left(Z_{B}^{+}\right)\right)^{2}+\left(\operatorname{div}\left(Z_{B}^{-}\right)\right)^{2} \neq 0$ in $S$, then, system (2) has at most $\ell$ limit cycles in $S$. We say that $B^{ \pm}$is a $\mathcal{C}^{1}$-Dulac piecewise function for system (2). Moreover, if $B^{ \pm}$is continuous on $\Sigma$ and $\operatorname{sgn} B^{+}=\operatorname{sgn} B^{-}$in $S$, then each limit cycle is hyperbolic and its stability is given by the sign of $B^{ \pm} \cdot \operatorname{div}\left(Z_{B}^{ \pm}\right)$over it.

In the following applications of the above theorem, we prove that at most one crossing limit cycle can appear. All of them can be considered in the Liénard class.

Proposition 1.3. Consider, for $\beta>0$, the piecewise differential system

$$
Z^{ \pm}=\left\{\begin{array}{l}
\dot{x}=y \\
\dot{y}=\beta\left(\alpha^{ \pm}-x\right)-\lambda^{ \pm} y
\end{array}\right.
$$

defined in $\Sigma^{ \pm}=\{(x, y): \pm x>0\}$. Then,

(a) when $\lambda^{+} \lambda^{-} \geq 0$ and $\left(\lambda^{+}\right)^{2}+\left(\lambda^{-}\right)^{2} \neq 0$, system (4) has no limit cycles,

(b) when $\lambda^{+} \lambda^{-}<0$ and $\left(\alpha^{+}\right)^{2}+\left(\alpha^{-}\right)^{2} \neq 0$ then, if $\alpha^{ \pm} \geq 0$ and $\left(\lambda^{+}\right)^{2}-4 \beta<0$ or $\alpha^{ \pm} \leq 0$ and $\left(\lambda^{-}\right)^{2}-4 \beta<0$, system (4) has at most one limit cycle being

$$
B_{1}^{ \pm}=\left(y^{2}+\beta\left(x^{2}-2 \alpha^{ \pm} x\right)+\lambda^{ \pm} x y\right)^{-1}
$$

$a \mathcal{C}^{1}$-Dulac piecewise function, otherwise it has no limit cycles. Moreover, when the limit cycle exists it is hyperbolic and stable (resp. unstable) when $\lambda^{+} \alpha^{+}<0$ or $\lambda^{+} \alpha^{-}<0$ (resp. $\lambda^{+} \alpha^{+}>0$ or $\left.\lambda^{+} \alpha^{-}>0\right)$. 
This problem, taking $\beta=\varepsilon$ small enough, $\alpha^{+}=\alpha^{-}=a$, and $\lambda^{ \pm}=k^{ \pm}$, is also considered in [19]. The uniqueness of limit cycle follows from the continuity of the piecewise vector field, see [22]. Moreover, a classification of all phase portraits is also obtained. We remark that, our generalized system (4) is only $\Sigma_{P^{-}}$continuous and the original proof does not work.

The next application is a piecewise generalization of the classical van der Pol oscillator, which is a dynamical system which includes a non-linear damping term. Under some conditions on the parameters, the Dulac function discovered by Cherkas (see $[4,5]$ ) can also be used here to prove the uniqueness of limit cycle. We provide also a partial result about the bifurcation diagram of the phase portraits in the Poincaré disk. More details on this theory can be found in [8].

Proposition 1.4. Consider the piecewise system

$$
Z^{ \pm}=\left\{\begin{array}{l}
\dot{x}=y, \\
\dot{y}=-x+\lambda^{ \pm}\left(1-x^{2}\right) y,
\end{array}\right.
$$

defined in $\Sigma^{ \pm}=\{(x, y): \pm x>0\}$. Then, when $\lambda^{+} \lambda^{-} \geq 0$ and $\left(\lambda^{+}\right)^{2}+\left(\lambda^{-}\right)^{2} \neq 0$, system (6) has exactly one limit cycle, which is hyperbolic and stable (resp. unstable) when $\lambda^{+}+\lambda^{-}>0$ (resp. $\lambda^{+}+\lambda^{-}<0$ ), with the associated $\mathcal{C}^{1}$-Dulac piecewise function

$$
B_{2}^{ \pm}=\left(x^{2}+y^{2}-1\right)^{-1 / 2} \text {. }
$$

Moreover, the phase portraits in the Poincaré disk are topologically conjugated to Figure 3 (left) when $\lambda^{+} \lambda^{-}>0$ or to Figure 3 (right) when $\lambda^{+} \lambda^{-}=0$, but $\left(\lambda^{+}\right)^{2}+\left(\lambda^{-}\right)^{2} \neq 0$.
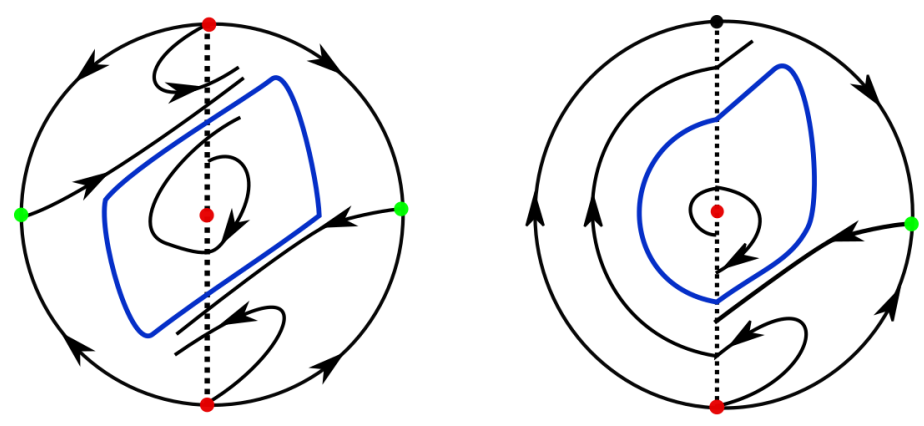

Figure 3. Phase portraits in the Poincaré disk for system (6)

The next differential system is also written in the classical Liénard form but being $F$ in (1) a rational function instead of a polynomial, as in the previous results. Here we also provide a Dulac function to prove the uniqueness of the limit cycle.

Proposition 1.5. Consider the rational piecewise system

$$
Z^{ \pm}=\left\{\begin{array}{l}
\dot{x}=y-\frac{x\left(x^{2}+\lambda^{ \pm}\right)}{(x \pm 1)^{2}+1} \\
\dot{y}=-x
\end{array}\right.
$$

defined in $\Sigma^{ \pm}=\{(x, y): \pm x>0\}$. Then, when $\lambda^{ \pm}<0$, system (8) has exactly one limit cycle, which is hyperbolic and stable, with the $\mathcal{C}^{1}$-Dulac piecewise function

$$
B_{3}^{ \pm}=\left(y^{2}-\frac{\left(x^{2}+\lambda^{ \pm}\right)}{(x \pm 1)^{2}+1} x y+x^{2}\right)^{-1} .
$$


We remark that, in Proposition 1.4, the function $B_{2}^{ \pm}$is, in fact, a Dulac function if we consider each system (6) separately but in the full plane. This is not the case for Propositions 1.3 and 1.5. But both together, each one considered in a different semiplane, define a $\mathcal{C}^{1}$-Dulac piecewise function for systems (4) and (8), respectively.

This paper is structured as follows, Section 2 is devoted to study the properties such that the Green's Theorem applies for piecewise vector fields and how the stability of a period orbit can be computed, because the classical divergence Theorem does not apply. Consequently, the definition of $\Sigma_{P}$-continuous piecewise differential systems has been necessary to be introduced. Furthermore, we also provide the proof of Theorem 1.2. In the rest of the paper, we prove the uniqueness of limit cycles for the Liénard families described above. In Section 3, we prove the statements of Propositions 1.3 and 1.5. In last Section 4, besides proving Proposition 1.4, we study the bifurcation diagram of the phase portraits of system (6) in the Poincaré disc.

\section{An extension of the BendiXson-Dulac Theory}

This section is devoted to the proof of Theorem 1.2, a generalization of the classical Bendixson-Dulac result on the non-existence and uniqueness of periodic orbits for piecewise vector fields. We will use the Green's Theorem on domains with smooth and piecewise smooth boundaries.

We start recalling the Bendixson and Dulac Theorems. See for example the textbooks $[5,8]$.

Theorem 2.1. Let $Z$ be a $\mathcal{C}^{1}$-planar vector field defined in some open simply connected region $S \subset \mathbb{R}^{2}$, such that

$$
\left.\operatorname{div}(Z)\right|_{S} \geq 0 \quad(\text { or } \leq 0)
$$

and vanishing only on a set of zero Lebesgue measure. Then, it has no limit cycles contained in $S$.

Theorem 2.2. Let $Z$ be a $\mathcal{C}^{1}$-planar vector field defined in some open simply connected region $S \subset \mathbb{R}^{2}$. If there exists a $\mathcal{C}^{1}$-Dulac function $B: S \rightarrow \mathbb{R}$, such that

$$
\left.\operatorname{div}(B Z)\right|_{S} \geq 0 \quad(\text { or } \leq 0),
$$

vanishing only on a set of zero Lebesgue measure, then $Z$ has no limit cycles contained in the region $S$.

In the above two results, the cases with null divergence are not considered because the vectors fields are integrable and have no limit cycles. In this special case, the function $B$ is called an inverse integrating factor. Later, we will show that this is not the case in the piecewise world.

The above two results can be extended to $\ell$-connected regions. For a proof of the next result see $[12,13]$.

Theorem 2.3. Let $Z$ be a $\mathcal{C}^{1}$-planar vector field defined in some $S \subset \mathbb{R}^{2}$ l-connected region. If there exists a $\mathcal{C}^{1}$-Dulac function $B: S \rightarrow \mathbb{R}$ such that in this region it satisfies condition (10), then $Z$ has at most $\ell$ limit cycles in $S$.

It is well-known that, for planar analytical differential systems, the integral of the divergence along a periodic orbit determines its stability, see [8]. But, for planar piecewise differential systems, this property cannot be used for crossing limit cycles. This is the main difference between these areas of research.

Let $\gamma=\gamma^{+} \cup \gamma^{-}$be a crossing periodic orbit passing through $q^{ \pm} \in \Sigma$, with $\gamma^{ \pm}$two solutions of $Z^{ \pm}$. If $\tau_{0}, \tau_{1} \subset \Sigma$ are transversal sections to $\gamma$ at $q^{+}$and $q^{-}$, respectively, then 
the derivative of the Poincaré map at $q=q^{+}$is given by

$$
\Pi^{\prime}(q)=\Gamma\left(Z^{ \pm}, h\right) \exp \left(\int_{\gamma^{+}} \operatorname{div} Z^{+}+\int_{\gamma^{-}} \operatorname{div} Z^{-}\right),
$$

where

see [24].

$$
\Gamma\left(Z^{ \pm}, h\right)=\frac{Z^{+}\left(q^{+}\right) \cdot \nabla h\left(q^{+}\right)}{Z^{-}\left(q^{+}\right) \cdot \nabla h\left(q^{+}\right)} \frac{Z^{-}\left(q^{-}\right) \cdot \nabla h\left(q^{-}\right)}{Z^{+}\left(q^{-}\right) \cdot \nabla h\left(q^{-}\right)},
$$

As a simple application of the above stability formula, we can check that the system

$$
\begin{aligned}
& Z^{+}:(\dot{x}, \dot{y})=\left(-3 y^{2}+8 y-4,-1\right), \\
& Z^{-}:(\dot{x}, \dot{y})=(y-a,-x),
\end{aligned}
$$

defined in $\Sigma^{ \pm}=\left\{(x, y) \in \mathbb{R}^{2}: \pm x \geq 0\right\}$, and studied in [3], has a unique stable crossing limit cycle when $2 / 3<a<1$. Straightforward computations shows that the limit cycle passes though the points $q^{ \pm}=\left(0, \mu^{ \pm}\right)$with $\mu^{ \pm}=a \pm \sqrt{3 a^{2}+8 a-4}$ and it can be explicitly written as

$$
\begin{cases}x^{2}+y^{2}-2 a y+4(a-1)^{2}=0 & \text { for } x<0 \\ y^{3}-4 y^{2}-x+4 y+8(a-2)(a-1)^{2}=0 & \text { for } x>0\end{cases}
$$

Moreover, system (13) has null divergence, but it exhibits an stable limit cycle because, by (11),

$$
\Pi^{\prime}(q)=\Pi^{\prime}\left(q^{+}\right)=\Gamma\left(Z^{ \pm}, h\right)=\frac{3 a^{2}-8 a+6+(3 a-4) \sqrt{3 a^{2}+8 a-4}}{2(a-1)(3 a-5)}<1 .
$$

In this work, we are interested in $\Sigma_{P}$-continuous piecewise differential systems. They satisfy, by Definition 1.1, that the derivative of the Poincaré map only depends on the integral of the divergence along a crossing periodic orbit. That is, $\Gamma\left(Z^{ \pm}, h\right)=1$, see (12).

Proof of Theorem 1.2. We will only prove the non-existence case and when system (2) has at most one limit cycle. The other cases follow similarly.

First, consider $B^{ \pm}$a $\mathcal{C}^{1}$-Dulac piecewise function defined in $S$, a 0 -connected region, see Figure 4 left. We will do a proof by contradiction. Assume that $\gamma^{ \pm}$is a crossing periodic orbit passing through the points $q^{ \pm} \in \Sigma$. We denote by $L$ the segment between them and by $A^{ \pm}$the interior of $\gamma^{ \pm}$. So, applying the Green's Theorem to $\left(Z_{B}^{ \pm}\right)^{\perp}$ (orthogonal to system (3)), we have

$$
\int_{L^{ \pm}}\left(-(B Y)^{ \pm} d x+(B X)^{ \pm} d y\right)=\iint_{A^{ \pm}}\left(\frac{\partial(B X)^{ \pm}}{\partial x}+\frac{\partial(B Y)^{ \pm}}{\partial y}\right) d x d y .
$$

Where the line integral on $\gamma^{ \pm}$vanishes, because it is a solution of the vector field $Z_{B}^{ \pm}$. We have denoted by $L^{ \pm}$the segment $L$ such that the boundary path of $A^{ \pm}$is clock-wise

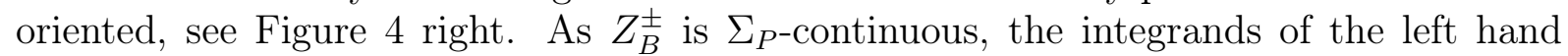
side of equalities (14) coincide, but $L^{ \pm}$have opposite orientation. Hence, adding both equalities, we get

$$
0=\int_{A^{+}} \operatorname{div}\left(Z_{B}^{+}\right) d x d y+\int_{A^{-}} \operatorname{div}\left(Z_{B}^{-}\right) d x d y
$$

Therefore, by hypotheses on the divergence of $Z_{B}^{ \pm}$we have a contradiction.

Second, we consider the system (3) with a $\mathcal{C}^{1}$-Dulac piecewise function $B^{ \pm}$defined in the 1-connected region $S$. The proof follows by contradiction assuming that there are two crossing periodic orbits, $\gamma_{i}^{ \pm}$for $i=1,2$, arguing similarly as the previous case, see Figure 5 left. The key point is, as above, the selection of a good oriented path defined 

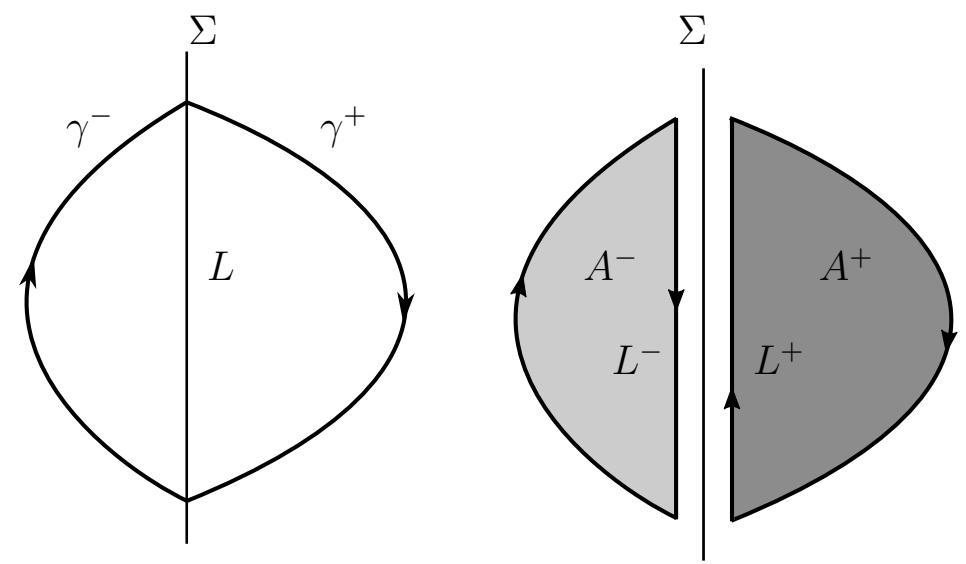

FIGURE 4. The regions and oriented paths chosen to apply Green's Theorem for one crossing periodic orbit.

in the boundary of the annular region $A^{ \pm}$. See Figure 5 right. Here, the $\Sigma_{P^{-c o n t i n u i t y ~} \text { is }}$ also necessary in order that the integral over $L_{i}^{+}$is compensated by the integral over $L_{i}^{-}$, for $i=1,2$. We notice that the integral over the other pieces of the boundary vanishes because the curves $\gamma_{i}^{ \pm}$are defined by solutions of the corresponding differential systems.
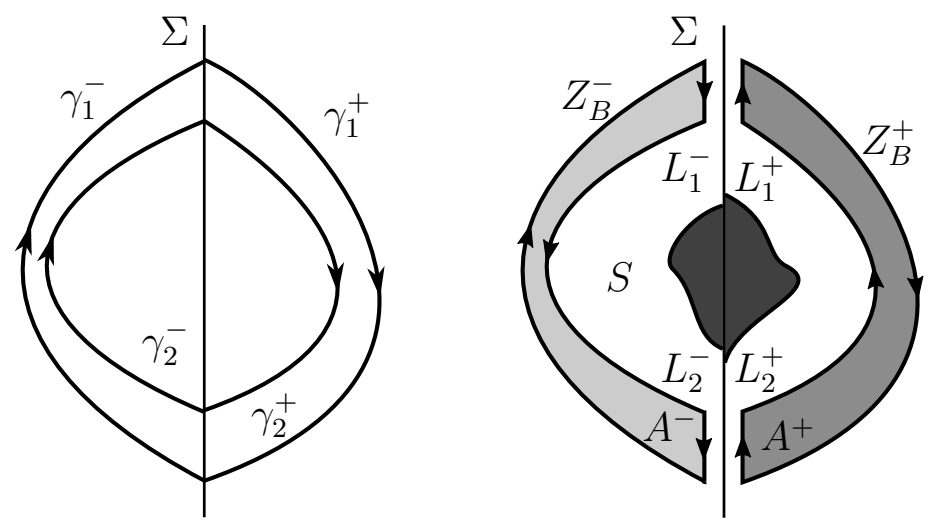

FiguRE 5. The regions and oriented paths chosen to apply Green's Theorem for two crossing periodic orbits.

Finally, we prove the hyperbolicity and stability properties of each limit cycle, $\gamma^{ \pm}$, from expression (11), showing that the integral of the divergence over it is non zero. This follows from the equality

$$
\operatorname{div}\left(Z^{ \pm}\right)=\frac{\operatorname{div}\left(Z_{B^{ \pm}}^{ \pm}\right)}{B^{ \pm}}-\frac{\frac{\partial B^{ \pm}}{\partial x} X^{ \pm}+\frac{\partial B^{ \pm}}{\partial y} Y^{ \pm}}{B^{ \pm}}
$$

and the fact that the second summand, as in [13], writes $\frac{d}{d t}\left(\log \left|B^{ \pm}(x(t), y(t))\right|\right)$. It is important to remark that we have used that the functions $B^{ \pm}$are continuous on $\Sigma$.

\section{THE LINEAR AND RATIONAL FAMILIES}

This section is devoted to prove Propositions 1.3 and 1.5. These results study two different piecewise differential systems in the Liénard class. The first, which is a linear, is a generalization of the continuous family introduced in [19]. We provide conditions on the parameter space such that the system has no limit cycles or it has at most one. For 
the second, which the nonlinearities are defined by rational functions, we can proof the existence of a unique limit cycle in a region of the parameter space.

The following technical lemma shows the condition about $\Sigma_{P}$-continuity on piecewise vector fields when the separation line is the $x$-axis. The proof is straightforward.

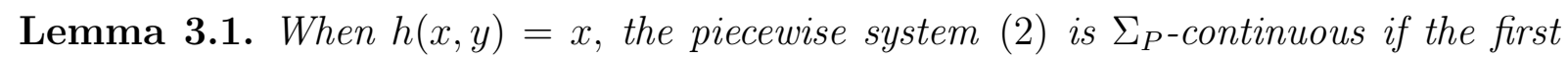
component of the associated vector field coincides on both sides, i.e. $X^{+}(0, y)=X^{-}(0, y)$.

Next proof is a direct application of Theorem 1.2. Only for the second statement we need to find a $\mathcal{C}^{1}$-Dulac piecewise function.

Proof of Proposition 1.3. (a) System (4) is defined in $\mathbb{R}^{2}$, that is a simple connected region,

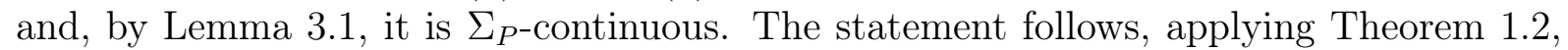
because the sign of the divergence, $\operatorname{div}\left(Z^{ \pm}\right)=-\lambda^{ \pm}$, coincides in both regions $\Sigma^{ \pm}$.

(b) This statement will be proved in three steps. In the first, we prove that a periodic orbit of (4) can not cross the pieces of the curves $\left\{\Lambda^{ \pm}(x, y)=0\right\} \cap \Sigma^{ \pm}$, where

$$
\Lambda^{ \pm}(x, y)=\left(B_{1}(x, y)\right)^{-1}=y^{2}+\lambda^{ \pm} x y+\beta\left(x^{2}-2 \alpha^{ \pm} x\right)
$$

is defined from (5). Because, these curves are without contact with the vector field associated to system (4). Then, the regions where the limit cycles can exist are completely determined. In the second, we discuss the different kind of regions defined by the zero level curves $\left\{\Lambda^{ \pm}(x, y)=0\right\}$. More concretely, we obtain only 0-connected or 1-connected regions. Finally, the proof finishes applying Theorem 1.2.

It is not restrictive to assume $\lambda^{+}>0, \lambda^{-}<0, \alpha^{+} \geq 0$ and $\alpha^{-}>0$. Because, the other cases can be moved to it, doing in system (4) the next changes of variables: (i) $(x, y, t) \rightarrow(-x, y,-t)$ when $\lambda^{+}>0, \lambda^{-}<0, \alpha^{+} \leq 0$ (ii) $(x, y, t) \rightarrow(x,-y,-t)$, when $\lambda^{+}<0, \lambda^{-}>0, \alpha^{+} \geq 0$, or (iii) $(x, y, t) \rightarrow(-x,-y, t)$ when $\lambda^{+}<0, \lambda^{-}>0, \alpha^{+} \leq 0$.

We notice that, with these sign assumptions, the divergence has different sign in regions $\Sigma^{ \pm}$, see Figure 6 left.

In the first step, using the notation (2) for system (4) and considering $\psi_{1}^{ \pm}(x)=\lambda^{ \pm} \beta \alpha^{ \pm} x$, we have that

$$
\begin{aligned}
\left.\left(\frac{\partial \Lambda^{ \pm}}{\partial x} X^{ \pm}+\frac{\partial \Lambda^{ \pm}}{\partial y} Y^{ \pm}\right)\right|_{\Lambda^{ \pm}=0} & =\left.\left(\frac{\partial \Lambda^{ \pm}}{\partial x} X^{ \pm}+\frac{\partial \Lambda^{ \pm}}{\partial y} Y^{ \pm}+\lambda^{ \pm} \Lambda^{ \pm}\right)\right|_{\Lambda^{ \pm}=0} \\
& =\left.\left(-\lambda^{ \pm} \beta \alpha^{ \pm} x\right)\right|_{\Lambda^{ \pm}=0} \leq 0 .
\end{aligned}
$$

Because, clearly, $\psi_{1}^{ \pm}$is a nonnegative function, see Figure 6 right. Then, the zero level
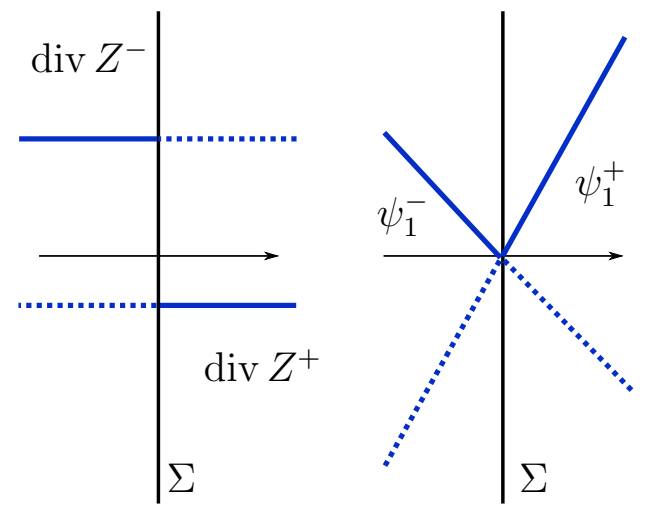

Figure 6. Plots of the sign of the divergence of system (4) (left) and the function $\psi_{1}^{ \pm}(x)$ (right) which appear in $\operatorname{div}\left(Z_{B_{1}}^{ \pm}\right)$. 
curve $\left\{\Lambda^{ \pm}(x, y)=0\right\}$ is without contact for the vector field associated to system (4), when $\alpha^{+} \neq 0$, or it contains solutions, when $\alpha^{+}=0$. In this last situation we refer the reader to the work [15].

In the second step, we study the draws of the pieces of the conics $\left\{\Lambda^{ \pm}(x, y)=0\right\}$, for $\Lambda^{ \pm}$in (15), when they remain on $\Sigma^{ \pm}$.

When $\alpha^{+}>0$, the zero level curve of $\Lambda^{+}$, is a nondegenerate conic: a hyperbola $\left(\left(\lambda^{+}\right)^{2}-4 \beta>0\right)$, a parabola $\left(\left(\lambda^{+}\right)^{2}-4 \beta=0\right)$ or an ellipse $\left(\left(\lambda^{+}\right)^{2}-4 \beta>0\right)$. Moreover, it has a branch passing through the origin as $2 \alpha^{+} \beta x-y^{2}+O\left(y^{3}\right)=0$ and another at the point $\left(2 \alpha^{+}, 0\right)$. When $\alpha^{+}=0$, the conic degenerates to two straight lines $\left(\left(\lambda^{+}\right)^{2}-4 \beta>0\right)$, to a double straight line $\left(\left(\lambda^{+}\right)^{2}-4 \beta=0\right)$ or to a point $\left(\left(\lambda^{+}\right)^{2}-4 \beta>0\right)$. Additionally, the origin is the unique intersection point with the coordinates axis. Finally, when $\left(\lambda^{+}\right)^{2}-4 \beta \geq 0$, as $-\lambda^{+} \pm \sqrt{\left(\lambda^{+}\right)^{2}-4 \beta}<0$, because $\lambda^{+}>0$, the branches of the conic arrive to infinity in the second or fourth quadrant. Consequently, all the possible the zero level curves are depicted in Figure 7 (right). The zero level curves of $\Lambda^{-}$, can be drawn, doing a symmetry with respect to the $x$-axis, from the previous study, because $\lambda^{-}<0$, instead of $\lambda^{+}>0$. See Figure 7 (left). So, we can see, doing a case by case study, that $\mathbb{R}^{2} \backslash\left(\left(\left\{\Lambda^{-}=\right.\right.\right.$

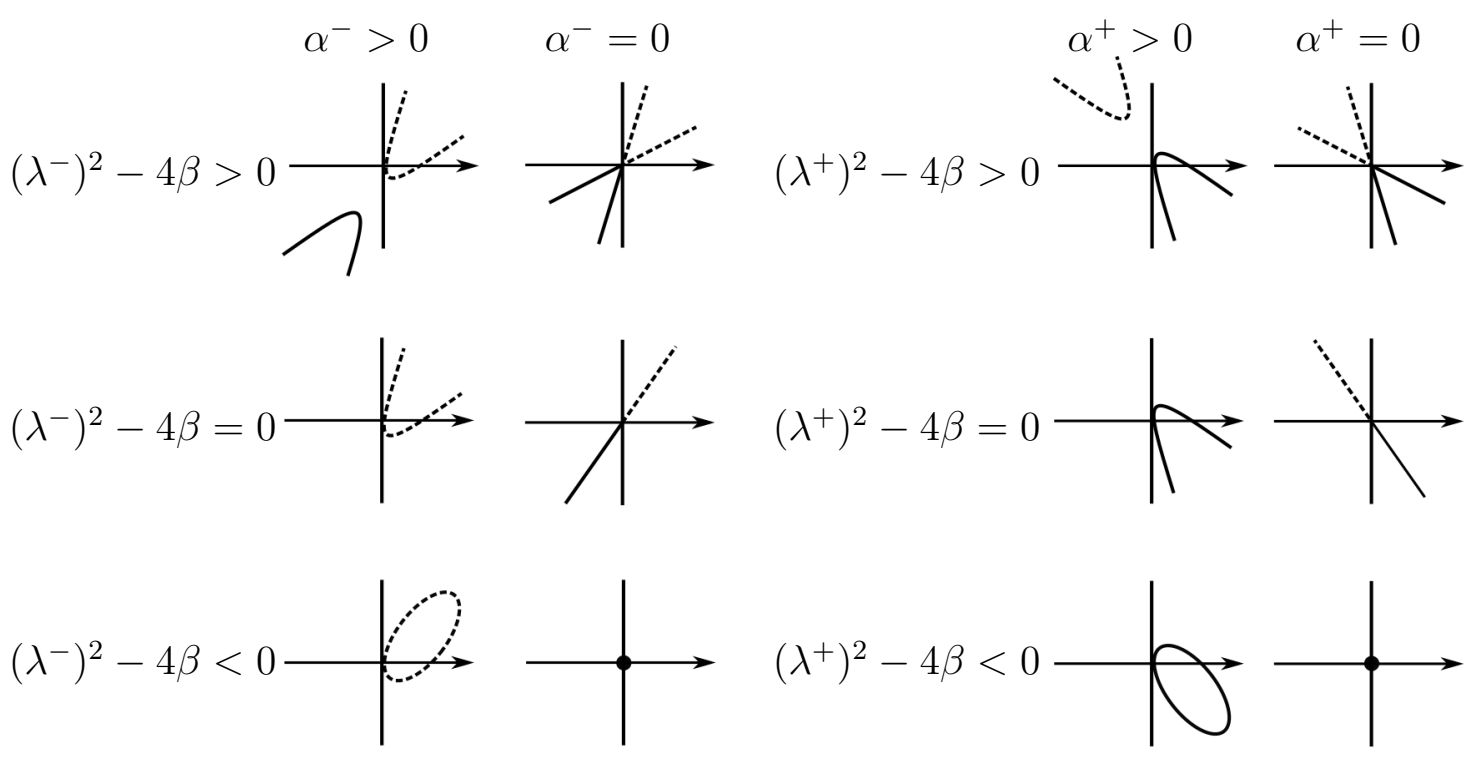

Figure 7. The sets, for $\lambda^{-}<0$ and $\lambda^{+}>0$, where the piecewise Dulac function (5) is not well defined. The branches are depicted in continuous (visible) and dashed (invisible) lines in the respective $\Sigma^{ \pm}$zones.

$\left.\left.0\} \cap \Sigma^{-}\right) \cup\left(\left\{\Lambda^{+}=0\right\} \cap \Sigma^{+}\right)\right)$is formed only by 0 -connected or 1-connected regions. In fact, only when $\left(\lambda^{+}\right)^{2}-4 \beta<0$ we have 1 -connected regions.

The last step follows directly computing, using $(16), \operatorname{div}\left(Z_{B_{1}}^{ \pm}\right)=\psi_{1}^{ \pm}(x) B_{1}^{ \pm}(x, y)^{2}$ and using the without contact property together with Theorem 1.2.

We only illustrate two different situations:

- When $\lambda^{-}=-1, \lambda^{+}=2, \alpha^{+}=2, \alpha^{-}=1$, and $\beta=1 / 2$, the zero level curve $\left\{\Lambda^{-}=0\right\}$ is an ellipse (totally contained in $\Sigma^{+}$) and $\left\{\Lambda^{+}=0\right\}$ is a hyperbola. Hence the set $\left\{\Lambda^{-}=0\right\} \cap \Sigma^{-}$is empty and the set $\left\{\Lambda^{+}=0\right\} \cap \Sigma^{+}$is a branch of the hyperbola. See the drawing in Figure 8 (left). Clearly, the region $\mathbb{R}^{2} \backslash\left(\left(\left\{\Lambda^{-}=0\right\} \cap \Sigma^{-}\right) \cup\left(\left\{\Lambda^{+}=0\right\} \cap \Sigma^{+}\right)\right)$ is defined by only two 0 -connected regions. Hence we have no limit cycles.

- When $\lambda^{-}=-2, \lambda^{+}=1 / 8, \alpha^{ \pm}=1$, and $\beta=1 / 10$, the zero level curve $\left\{\Lambda^{-}=0\right\}$ is a hyperbola and $\left\{\Lambda^{+}=0\right\}$ is an ellipse. See the drawing in Figure 8 (right). So, the region $\mathbb{R}^{2} \backslash\left(\left(\left\{\Lambda^{-}=0\right\} \cap \Sigma^{-}\right) \cup\left(\left\{\Lambda^{+}=0\right\} \cap \Sigma^{+}\right)\right)$is defined by three regions, two 0 -connected 
and one 1-connected. Hence, we have at most one limit cycle. The existence, for these values of the parameters, is proved in [19].
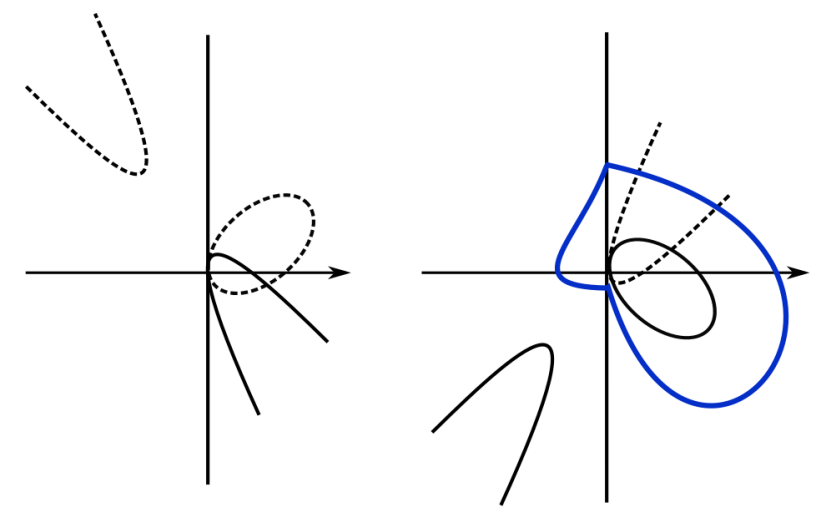

Figure 8. Two different situations for system (4) with only 0-connected regions (left) or having a 1-connected one (right).

Finally, as the function $B_{1}^{ \pm}$is continuous over $\Sigma$, with the sign assumptions for $\alpha^{ \pm}$and $\lambda^{ \pm}$, Theorem 1.2 proves the hyperbolicity and unstability properties.

Before the proof of Proposition 1.5, we show the unstability of the origin and infinity of system (8).

Proposition 3.2. Let system (8). If $\lambda^{ \pm}<0$, then the origin is the unique singular point which is unstable. Moreover, also the infinity is unstable. So system (8) has at least a limit cycle.

Proof. The origin is the unique equilibrium point of systems (8) when they are considered in the full $\mathbb{R}^{2}$. In fact, for both, it is unstable because the eigenvalues of the differential matrices at the origin, $\left(-\lambda \pm \sqrt{\lambda^{2}-16}\right) / 4$ for $\lambda=\lambda^{ \pm}$, have positive real parts. See more details on the stability of equilibrium points in piecewise differential systems in [23].

The stability of infinity can be studied with the change of variables defined in a neighborhood of the infinity but not on it: $(x, y)=\left(R^{-1} \cos \theta, R^{-1} \sin \theta\right)$. Then, the derivatives $\dot{R}$ and $\dot{\theta}$ are trigonometric rational functions having the same denominator $(\cos \theta+R)^{2}+R^{2}$. Which is always positive in our domain, $R>0$. Then, the linear part of system (8) becomes $d R / d \theta=h(\theta) R$ with $h(\theta)=\cos ^{2} \theta /(\cos \theta \sin \theta-1)$. Notice that it is the same in both sides $\Sigma^{ \pm}$, and does not depend on the parameters. As $R(\theta)$ is a decreasing function, because $-4 / 3 \leq h(\theta) \leq 0$, for any the initial condition $R(\pi / 2)=\rho$ we have $R(-3 \pi / 2)>\rho>R(-\pi / 2)$. Consequently, for both equations and also for the system considered in $\Sigma^{ \pm}$, the origin is an attracting focus. Hence, recovering the original variables, the infinity is unstable.

Proof of Proposition 1.5. Under the conditions of the statement, Proposition 3.2 guaranties the existence of a limit cycle. Only remains to prove that this limit cycle is unique. This is proved checking the hypotheses of Theorem 1.2 for system (8) when $\lambda^{ \pm}<0$, following the same procedure as the proof of Proposition 1.3(b). Hence, as the periodic orbits of (8) can not cross the pieces of the curves $\left\{\Lambda^{ \pm}(x, y)=0\right\} \cap \Sigma^{ \pm}$, we only check that this set defines a 1 -connected region and $\operatorname{div}\left(Z_{B_{3}}^{ \pm}\right)$have the same sign in $\Sigma^{ \pm}$. Here

$$
\Lambda^{ \pm}(x, y)=\left(B_{3}^{ \pm}(x, y)\right)^{-1}=y^{2}-\frac{\left(x^{2}+\lambda^{ \pm}\right)}{(x \pm 1)^{2}+1} x y+x^{2}
$$

is defined from (9). 
Straightforward computations show that the divergence

$$
\operatorname{div}\left(Z^{ \pm}\right)=\frac{-x^{4} \mp 4 x^{3}+\left(\lambda^{ \pm}-6\right) x^{2}-2 \lambda^{ \pm}}{\left((x \pm 1)^{2}+1\right)^{2}}
$$

is a rational function on $x$ that changes sign. Because the numerators, using the Descartes rule, have exactly one positive and one negative zeros.

In the first step, we compute

$$
\operatorname{div}\left(Z_{B_{3}}^{ \pm}\right)=\psi_{3}^{ \pm}(x) B_{3}^{ \pm}(x, y)^{2},
$$

with $\psi_{3}^{ \pm}(x)=2 x^{3} \varphi^{ \pm}(x) /\left((x \pm 1)^{2}+1\right)^{2}$ and $\varphi^{ \pm}(x)=\mp x^{2}+\left(\lambda^{ \pm}-2\right) x \pm \lambda^{ \pm}$. Clearly, $x \varphi^{+}(x)<0$ for $x>0$ because the leading coefficient of $\varphi^{+}$and the values $\varphi^{+}(0)$ and $\left(\varphi^{+}\right)^{\prime}(0)$ are negative. Similarly, $x \varphi^{-}(x)<0$ for $x<0$. Hence, $\operatorname{div}\left(Z_{B_{3}}^{ \pm}\right)<0$ in $\Sigma^{ \pm}$and it vanishes only on $x=0$. As the functions $B_{3}^{ \pm}$

In the second step, we study the piecewise curves $\left\{\Lambda^{ \pm}(x, y)=0\right\} \cap \Sigma^{ \pm}$. We only depict the zero level curve of $\Lambda^{+}$, in (17), the other can be obtained with the symmetric change $(x, y) \rightarrow(-x,-y)$, because $\Lambda^{-}(x, y)=\Lambda^{+}(-x,-y)$. We remark that this curve passes through the origin. We draw it from the plots of the two functions, obtained solving it as a quadratic polynomial in $y$,

$$
\eta_{ \pm}\left(\lambda^{+}\right)=\frac{x\left(x^{2}+\lambda^{+} \pm \sqrt{\Delta\left(x, \lambda^{+}\right)}\right)}{2\left((x+1)^{2}+1\right)},
$$

with

$$
\Delta\left(x, \lambda^{+}\right)=-\left(3 x^{2}+4 x+\lambda^{+}+4\right)\left((x+2)^{2}-\lambda^{+}\right) .
$$

As $\lambda^{+}<0$, the function $\Delta\left(x, \lambda^{+}\right)$is always negative when $-8 / 3<\lambda^{+}<0$ and it is positive for $x \in\left(\left(-2-\sqrt{-8-3 \lambda^{+}}\right) / 3,\left(-2+\sqrt{-8-3 \lambda^{+}}\right) / 3\right)$ when $\lambda^{+}<-8 / 3$. The graphics of $\eta_{ \pm}$ remain in $\Sigma^{+}$only when $-2+\sqrt{-8-3 \lambda^{+}}$is positive, that is, when $\lambda^{+}<-4$. Consequently, the level curve $\left\{\Lambda^{+}(x, y)=0\right\}$ intersects $\Sigma^{+}$only at the origin when $\lambda^{+} \in[-4,0)$ and at a curve topologically equivalent to a circle when $\lambda^{+}<-4$. The different draws of such situations can be seen in Figure 9 varying the value of $\lambda^{+}$.
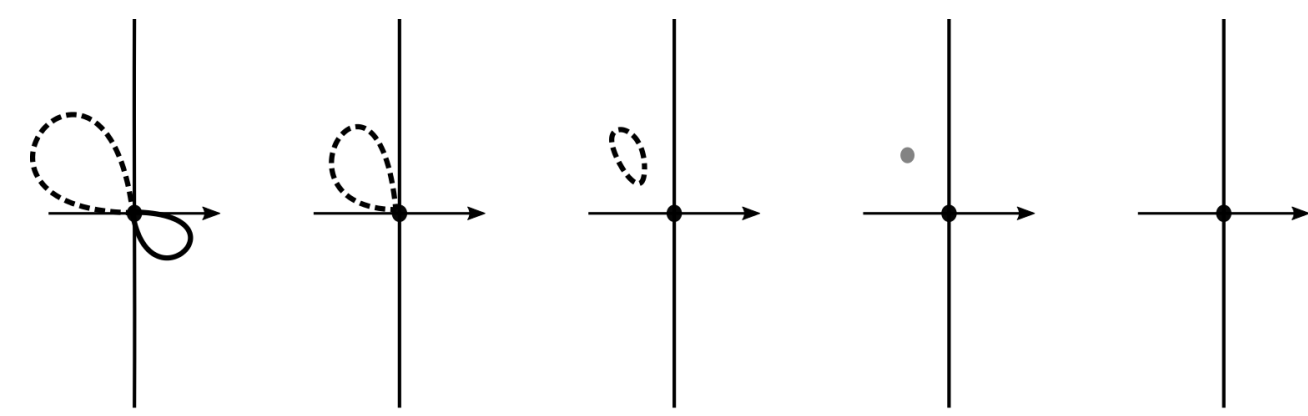

$$
\lambda^{+}<-4
$$

$$
\lambda^{+}=-4
$$$$
-4<\lambda^{+}<-\frac{8}{3}
$$$$
\lambda^{+}=-\frac{8}{3} \quad-\frac{8}{3}<\lambda^{+}<0
$$

Figure 9. The zero level curve of $\Lambda^{+}$in (17). The points in $\Sigma^{+}\left(\Sigma^{-}\right)$are depicted in continuous (dashed) line.

Finally, a case by case study, using the symmetry of the functions $\Lambda^{ \pm}$and the pictures in Figure 9, proves that all possible draws for the piecewise curves $\left\{\Lambda^{ \pm}(x, y)=0\right\} \cap \Sigma^{ \pm}$ define 1-connected regions. See Figure 10. 

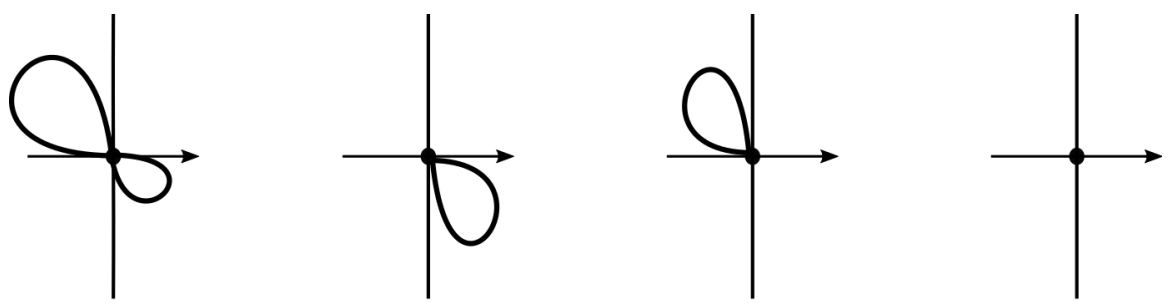

$$
\lambda^{ \pm}<-4
$$

$$
\begin{gathered}
-4 \leq \lambda^{-}<0 \\
\lambda^{+}<-4
\end{gathered}
$$

$$
-4 \leq \lambda^{+}<0
$$$$
-4 \leq \lambda^{ \pm}<0
$$

FiguRE 10. The 1-connected regions defined by the zero level curve of $\Lambda^{ \pm}$in (17).

\section{A Piecewise version of the Classical van DeR Pol oscillator}

The main result of this section, Proposition 1.4, only provides a partial bifurcation diagram of the phase portrait of system (6). A complete study depends on the proof of the existence of a bifurcation curve, $\Upsilon$, where the phase portrait exhibits a connection between two equilibrium points at infinity. This connection defines a heteroclinic cycle having two semihyperbolic saddles together with a degenerated equilibrium point. These degeneracies make the problem very difficult to be studied. All the results and numerical simulations done in this section are summarized in Figure 11. The phase portraits are done in the Poincaré disk for each vector fied, see [8].

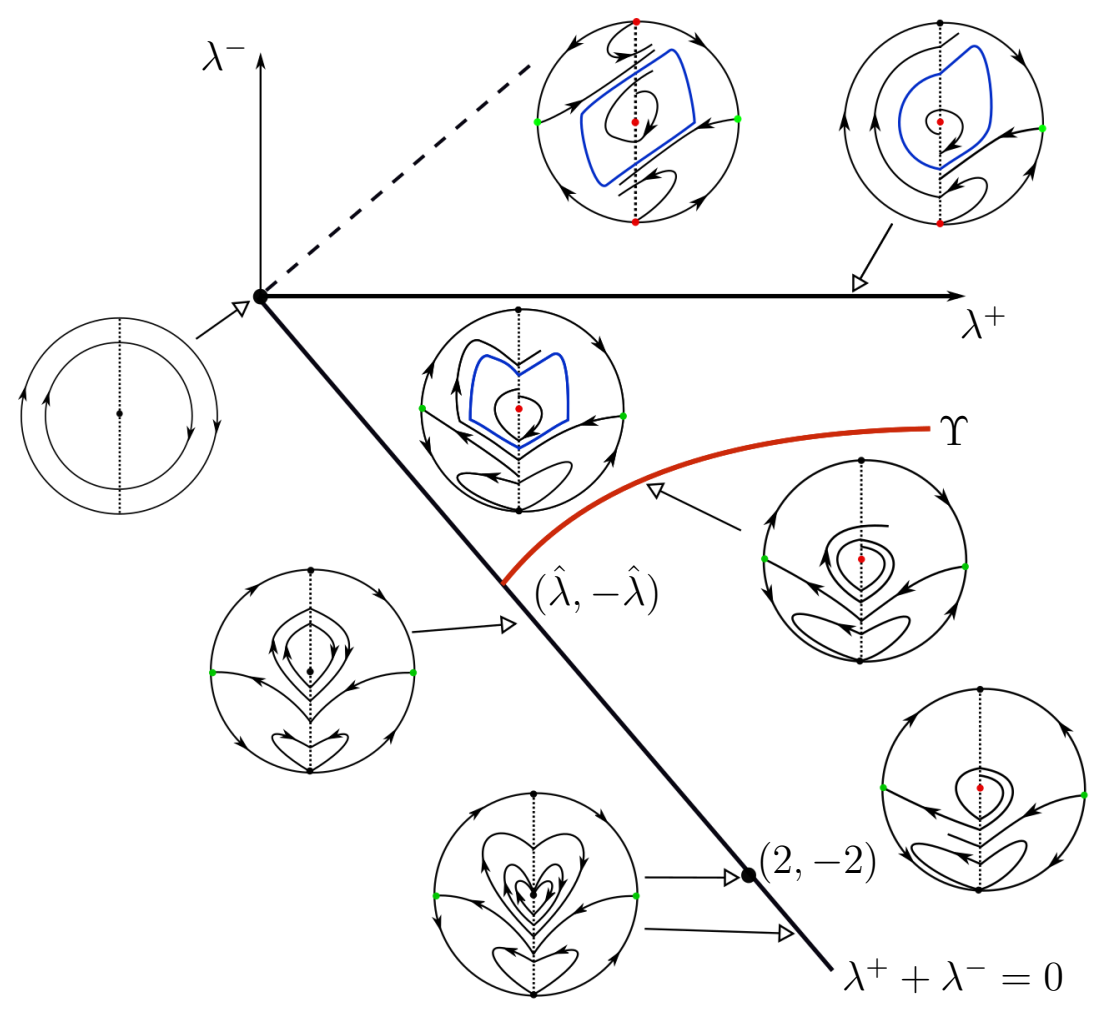

Figure 11. Portrait bifurcation of system (6).

For simplicity, all the results of this section are written for values $\left(\lambda^{+}, \lambda^{-}\right)$satisfying $\lambda^{+} \geq 0$ and $-\lambda^{+} \leq \lambda^{-} \leq \lambda^{+}$. With the symmetries given in Lemma 4.1 we can cover the full space of parameters. The local phase portrait of the equilibrium points at infinity is done in Lemma 4.2. System (6) has only one finite equilibrium point and its stability is given in Lemma 4.3. From all these technical results we can present the proof of the uniqueness of the limit cycle together with the global phase portraits provided 
by Proposition 1.4. This proves the bifurcation diagram depicted in Figure 11 in the region $\lambda^{+} \lambda^{-} \geq 0$. By a continuity argument this limit cycle remains near the positive $\lambda^{+}$-axis. This is done in Corollary 4.4. Proposition 4.5 proves the bifurcation diagram when system (6) has a center, that is, over the straight line $\lambda^{+}+\lambda^{-}=0$.

The difficulties described above to complete the bifurcation diagram (for $\lambda^{-}<0$ ) are illustrated in Proposition 4.6. This result together with Corollary 4.4 prove, by continuity, Corollary 4.7, which states the existence of at least one point $\left(\lambda^{+}, \lambda^{-}\right)$where the connection exists. Finally, we do some numerical simulations to present what we think that will be the complete bifurcation diagram of system (6) in this zone of the parameters. See also Figure 11.

Lemma 4.1. System (6) is invariant by the following changes of variables:

(i) $\left(x, y, t, \lambda^{-}, \lambda^{+}\right) \rightarrow\left(-x,-y, t, \lambda^{+}, \lambda^{-}\right)$.

(ii) $\left(x, y, t, \lambda^{-}, \lambda^{+}\right) \rightarrow\left(-x, y,-t,-\lambda^{+},-\lambda^{-}\right)$.

(iii) $\left(x, y, t, \lambda^{-}, \lambda^{+}\right) \rightarrow\left(x,-y,-t,-\lambda^{-},-\lambda^{+}\right)$.

Consequently, we can restrict our analysis to $\lambda^{+}>0$ and $-\lambda^{+} \leq \lambda^{-} \leq \lambda^{+}$.

Proof. The statement follows because, in the parameter space $\left(\lambda^{-}, \lambda^{+}\right) \in \mathbb{R}^{2}$, the points in the second and third quadrant move with the change (ii) to the fourth and first, respectively. Moreover, changes (i) and (iii) provides an extra symmetry with respect to the bisectors of the first and fourth quadrants, respectively.

Lemma 4.2. Let $\lambda^{+}>0$. Then, for $\lambda^{-} \in\left[-\lambda^{+}, \lambda^{+}\right]$, there are only three topologically conjugated local phase portraits of system (6) in a neighborhood of infinity. They are depicted in Figure 12.
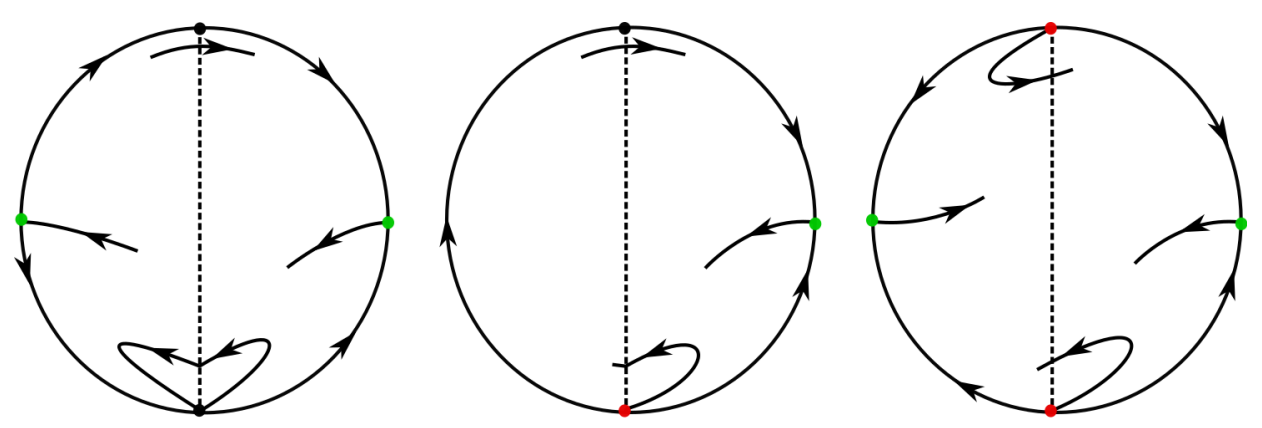

FIGURE 12. Local phase portraits of system (6) near the infinity when $\lambda^{+}>0$ and $\lambda^{-}<0$ (left), $\lambda^{-}=0$ (middle), and $\lambda^{-}>0$ (right).

Proof. (i) We start with the local study in the charts $\mathcal{U}_{1}$ and $\mathcal{V}_{1}$. We study both systems, for $\lambda^{+}$and $\lambda^{-}$, simultaneously denoting the parameter by $\lambda$, when they are non vanishing. With the change $(x, y, t)=\left(1 / v, u / v, v^{2} \tau\right)$, system (6), considered in full space, is transformed to

$$
\left\{\begin{array}{l}
u^{\prime}=-\lambda u+A(u, v), \\
v^{\prime}=B(u, v),
\end{array}\right.
$$

where $A(u, v)=\lambda u v^{2}-u^{2} v^{2}-v^{2}, B(u, v)=-v^{3} u$, and prime denotes the derivative respect to $\tau$. This system has a unique equilibrium point at the origin which is a semihyperbolic saddle. Because, by Theorem 2.19 in [8], the solution $u(v)=-v^{2} / \lambda-v^{4} / \lambda+O\left(v^{6}\right)$ of $-\lambda u+A(u, v)=0$ gets $B(u(v), v)=-v^{5} / \lambda+O\left(v^{7}\right)$. The local phase portraits depend on the sign of $\lambda$, see Figure 13. 

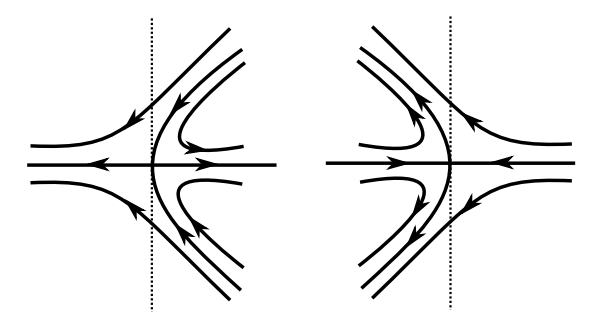

Figure 13. The local phase portrait of the origin of system (18) for $\lambda<0$ (left) and $\lambda>0$ (right).

Then, in the chart $\mathcal{U}_{1}$, as $\lambda^{+}$is postive, we have always the local phase portrait as in Figure 13 (right). The local phase portraits, in the chart $\mathcal{V}_{1}$, depend on the sign of $\lambda^{-}$. Being as in Figure 13 (left) or Figure 13 (right) for $\lambda^{-}<0$ or $\lambda^{-}>0$.

For the remaining case, $\lambda^{-}=0$, the system (6) has a global linear center at the origin. Then, the change to chart $\mathcal{V}_{1}$ is not necessary to be done.

With all the above properties, we obtain the local phase portraits depicted in Figure 12.

(ii) As above, we study both systems simultaneously using $\lambda$ instead of $\lambda^{+}$or $\lambda^{-}$.

By using the transformation $(x, y, t)=\left(u / v, 1 / v, v^{2} \tau\right)$ system (6) is transformed into the system

$$
\left\{\begin{array}{l}
u^{\prime}=\lambda\left(u^{3}-u v^{2}\right)+v^{2}+u^{2} v^{2}, \\
v^{\prime}=v\left(\lambda\left(u^{2}-v^{2}\right)+u v^{2}\right)
\end{array}\right.
$$

where the prime denotes the derivative respect to $\tau$. It is easy to check that this system has a unique degenerated equilibrium point at the origin. As it is a nonelementary singularity, we will need some directional blow-ups to prove that it is an attracting $(\lambda<0)$ or a repelling $(\lambda>0)$ node, see Figure 14. This is necessary because the differential matrix of (19) at the origin vanishes identically. More details about how the local phase portraits of such degenerated equilibrium points can be also found in [8]. As system (19) is invariant by the change $(u, v, t, \lambda) \rightarrow(-u, v,-t,-\lambda)$, we can restrict our study to $\lambda>0$.
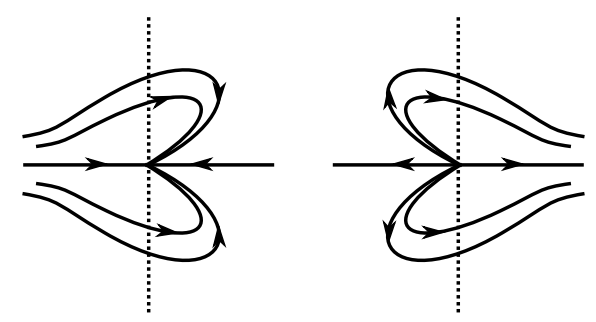

Figure 14. The local phase portrait of the origin of system (19) for $\lambda<0$ (left) or $\lambda>0$ (right).

With the weighted blow-up $(u, v) \rightarrow\left(u, p u^{2}\right)$ and rescaling time (dividing by $u^{2}$ ), system (19) becomes

$$
\left\{\begin{array}{l}
u^{\prime}=\lambda u+u^{2} p^{2}\left(1-\lambda u+u^{2}\right), \\
p^{\prime}=-\lambda p+u p^{3}\left(-2+\lambda u-u^{2}\right) .
\end{array}\right.
$$

This system has a unique equilibrium point, which is a saddle. The local phase portrait is given in Figure 15 (left). 

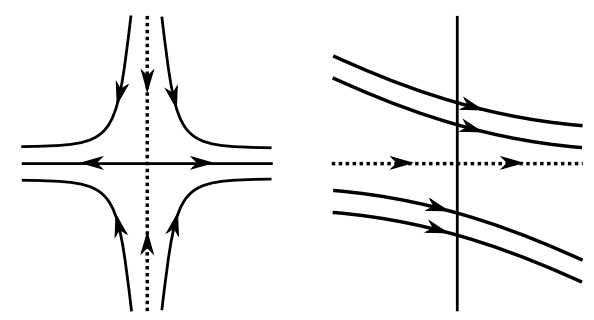

Figure 15. The local phase portrait of the origin of systems (20) and (21) for $\lambda>0$, respectively.

With the blow-up $(u, v) \rightarrow(q v, v)$ and rescaling time (dividing by $v$ ) the system (19) becomes

$$
\left\{\begin{array}{l}
q^{\prime}=1, \\
v^{\prime}=v^{2}\left(-\lambda+q v+\lambda q^{2}\right) .
\end{array}\right.
$$

Which has no singular points. The local phase portrait is given in Figure 15 (right). Finally, combining both local figures and using that $v=0$ is also a solution and it is the unique that leaves the origin, we can conclude that the origin of system (19) is a repelling node. The conclusion for $\lambda<0$ follows from the above commented symmetry. The solutions are depicted in Figure 14.

Lemma 4.3. The bifurcation diagram of the topologically different local phase portraits near the origin of system (6), when $\lambda^{+} \geq 0$ and $-\lambda^{+} \leq \lambda^{-} \leq \lambda^{+}$, is depicted in Figure 16. Moreover, when $\lambda^{+}>0$ and $\max \left(-2,-\lambda^{+}\right) \leq \lambda^{-} \leq \lambda^{+}$the origin is unstable.

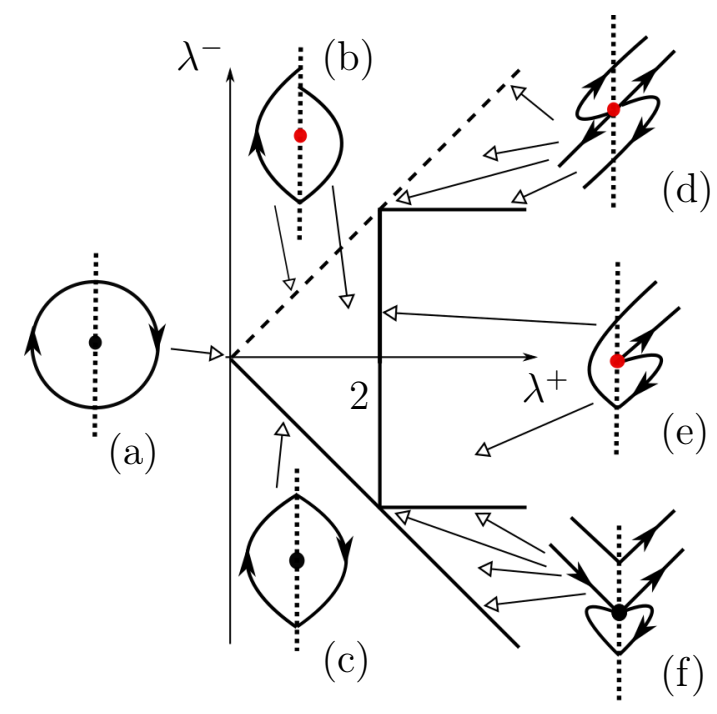

FiguRE 16. The different kinds of equilibrium points in the origin for the system (6).

Proof. We do a case by case study depending on the eigenvalues of the Jacobian matrices of each system $Z^{ \pm}$in (6) at the origin. They are

$$
\frac{\lambda \pm \sqrt{\lambda^{2}-4}}{2}
$$

for $\lambda=\lambda^{+}$and $\lambda=\lambda^{-}$. All cases finish gluing both local pictures in $Z^{ \pm}$. 
(a) When $\lambda^{ \pm}=0$ we have the same global linear center at the origin.

(b) When $0<\lambda^{+}<2$ and $-\lambda^{+}<\lambda^{-} \leq \lambda^{+}$we have foci in $Z^{ \pm}$. Then, as the singularities are of monodromic type we need to compute the first coefficient of the return map. That is, the first Lyapunov constant. From [6], it is

$$
L_{1}=\exp \left(\frac{\left(\lambda^{+}+\lambda^{-}\right) \pi}{\sqrt{4-\left(\lambda^{+}\right)^{2}} \sqrt{4-\left(\lambda^{-}\right)^{2}}}\right)-1 .
$$

Then, when $\lambda^{+}+\lambda^{-}>0$ the origin is a unstable focus.

(c) When $0<\lambda^{+}<2$ and $\lambda^{+}+\lambda^{-}=0$, as in the previous case, the origin is a monodromic point. Then, it is center because the system is symmetric with respect to $x=0$. That is, is reversible, or it remains unchanged with respect to the change $(x, y, t) \rightarrow(-x, y,-t)$.

(d) When $2 \leq \lambda^{+}$and $-2<\lambda^{-}<2$, system $Z^{-}$has a focus at the origin and system $Z^{+}$has a repelling node with both eigenvectors having positive slopes $\left(1,\left(\lambda^{+} \pm\right.\right.$ $\left.\sqrt{\left(\lambda^{+}\right)^{2}-4}\right) / 2$ ). Then, the origin is an unstable node.

(e) When $2 \leq \lambda^{+}$and $2 \leq \lambda^{-} \leq \lambda^{+}$, both systems $Z^{ \pm}$have repelling nodes at the origin with eigenvectors having positive slopes $\left(1,\left(\lambda^{+} \pm \sqrt{\left(\lambda^{+}\right)^{2}-4}\right) / 2\right)$ and $\left(1,\left(\lambda^{-} \pm\right.\right.$ $\left.\sqrt{\left(\lambda^{-}\right)^{2}-4}\right) / 2$ ). Then, the origin is also unstable.

(f) When $2 \leq \lambda^{+}$and $-\lambda^{+} \leq \lambda^{-} \leq-2$, system $Z^{+}$(resp. $Z^{-}$) has a repelling (resp. attracting) node at the origin with eigenvectors having positive (resp. negative) slopes $\left(1,\left(\lambda^{+} \pm \sqrt{\left(\lambda^{+}\right)^{2}-4}\right) / 2\right)\left(\operatorname{resp} .\left(1,\left(\lambda^{-} \pm \sqrt{\left(\lambda^{-}\right)^{2}-4}\right) / 2\right)\right)$. Then, we have a point having one hyperbolic and one elliptic sectors.

The above two technical lemmas together with the main result of generalization of the Bendixson-Dulac Theorem allow us to proof the main result of this section.

Proof of Proposition 1.4. Firstly, as we have commented in the introduction of this section and using Lemma 4.1, we can restrict our proof to values satisfying $\lambda^{+}>0, \lambda^{-} \geq 0$. Under these assumptions, the existence of at least one limit cycle follows from the stability of infinity and origin, because both are unstable. See Lemmas 4.2 and 4.3. This follows by the generalization of the Poincaré-Bendixson's Theorem to piecewise vector fields in $\mathbb{R}^{2}$. See [2].

Secondly, we prove the uniqueness of such periodic orbit. As $\operatorname{div} Z^{ \pm}$is positive in the region $S=\{-1<x<1\}$, from Theorem 1.2, the limit cycles can not be completely contained in $S$. In fact, they should contain the 1-connected region defined by the unit circle. Because, as in the proofs of Propositions 1.3 or 1.5, the periodic orbit of (4) can not cross the pieces of the curves $\{\Lambda(x, y)=0\} \cap \Sigma^{ \pm}$, where

$$
\Lambda^{ \pm}(x, y)=\left(B_{2}^{ \pm}(x, y)\right)^{-2}=x^{2}+y^{2}-1
$$

is defined from (7). We point out that they are without contact with the vector field associated to system (6). Moreover, $\operatorname{div}\left(Z_{B_{2}}^{ \pm}\right)$is negative when $x^{2}+y^{2}>1$. Then, using also Theorem 1.2, the limit cycle is unique. Moreover, as $B_{2}^{ \pm}$is continuous, it is hyperbolic and stable.

All the global phase portraits in the Poincare disk given in the statement, follow studying all the possible $\alpha$ and $\omega$ limit sets using the generalization of the Poincaré-Bendixson's Theorem. This is done combining the local phase portrait of the infinity and origin in Lemmas 4.2 and 4.3, and, finally, the uniqueness of the periodic orbit proved above.

Next result provides the existence of a limit cycle near the $\lambda^{+}$-axis. 
Corollary 4.4. For each $\lambda^{+}>0$, there exists $\varepsilon>0$ small enough such that system (6), for $\max \left(-\lambda^{+},-\varepsilon\right)<\lambda^{-}<0$, has at least one limit cycle, which is stable. Moreover, the phase portraits in the Poincaré disk is shown in Figure 17, when the limit cycle is unique.

Proof. For the conditions given in the statement, Lemma 4.3 provides the unstability of the origin. For $\lambda^{-}=0$, from Proposition 1.4, the $\omega$ limit set of the separatrix of the saddle at infinity for $Z^{+}$is the stable limit cycle. Using the behavior of infinity, for $\lambda^{-}<0$ small enough, given in Lemma 4.2, a continuity argument guaranties the existence of at least a periodic orbit.

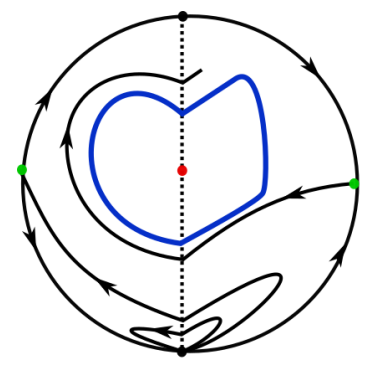

Figure 17. Phase portrait of system (6) for $\lambda^{+}>0$ and small enough $\lambda^{-}<0$ if the limit cycle is unique.

The values of $\left(\lambda^{+}, \lambda^{-}\right)$where system (6) exhibits a center is done in the following result.

Proposition 4.5. When $\lambda^{+} \geq 0$ and $\lambda^{+}+\lambda^{-}=0$ system (6) has a time-reversible center with respect to the $y$-axis. More concretely, when $\lambda^{+}=0$ it has a global linear center, when $0<\lambda^{+}<2$ the inner boundary is the origin which is monodromic, and when $2 \leq \lambda^{+}$the inner boundary is a degenerated equilibrium point having one hyperbolic and one elliptic sectors. Moreover, when $\lambda^{+} \neq 0$ there is a finite solution connecting two saddles at infinity that defines the finite part of the outer boundary. See the respective phase portraits in Figure 18.
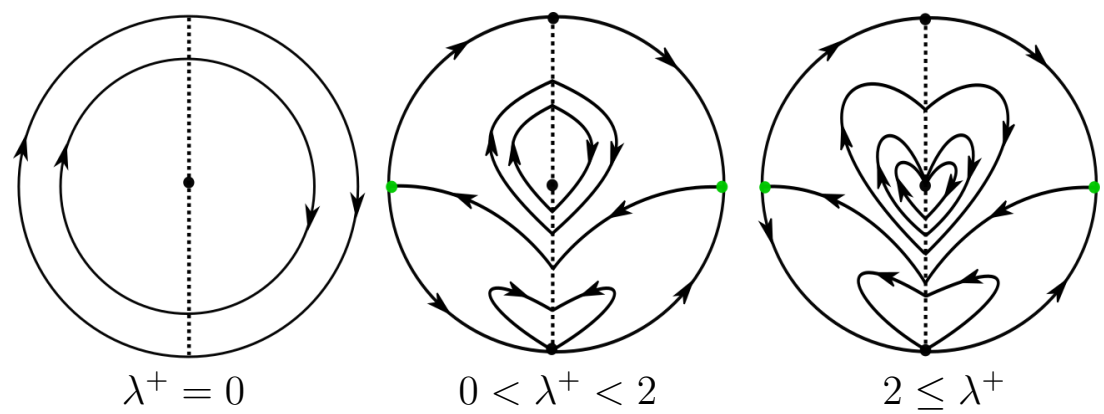

Figure 18. Phase portrait of system (6) $\lambda^{+}+\lambda^{-}=0$.

Proof. The time-reversibility property follows from the condition $\lambda^{+}+\lambda^{-}=0$ applying the change $(x, y, t) \rightarrow(-x, y,-t)$. Lemma 4.3 provides the local phase portraits of the origin. When $\lambda^{+}=0$ the local center at the origin is global because in both sides we have the same system. When $0<\lambda^{+}<2$ the origin is also a local center, see Figure 16(c) but when $2 \leq \lambda^{+}$the origin is a degenerate singularity with one hyperbolic and one elliptic sectors, see Figure 16(f). Adding the local phase portrait at infinity, see Figure 12 (left), the global picture follows, using the reversibility property, showing that the unstable separatrix of the saddle at infinity of $Z^{+}$crosses the negative $y$-axis. See Figure 18. In both cases, 
using Poincaré-Bendixson theorem in the Poincaré disk for piecewise vector fields, see [2], from the sign of the components of the vector field associated to system (6) and looking at infinity, any solution starting at a point on the positive $y$-axis should arrive to the negative $y$-axis crossing the positive $x$-axis. Then, also the unstable separatrix should do the same. From the pictures is clear that this separatrix defines a piece of the outer boundary.

Proposition 4.6. Consider system (6) with $\lambda^{+}=6$ and $\lambda^{-}=-4$. Let $\Gamma^{ \pm}$be the pieces of curves defined by the solutions corresponding to the unstable (resp. stable) separatrix of the origin of chart $\mathcal{U}_{1}$ (resp. $\mathcal{V}_{1}$ ) up to the first intersection with the $x$-axis. Then $\Gamma^{-} \cap\{x \leq 0\} \subset\left\{y-\Phi^{-}(x)>0\right\}$ and $\Gamma^{+} \cap\{x \geq 0\} \subset\left\{y-\Phi^{+}(x)<0\right\}$, see Figure 19. Where

$$
\Phi^{-}(x)=\left\{\begin{array}{ll}
\Phi_{1}^{-}(x) & \text { for }-\frac{11}{10} \leq x \leq 0, \\
\Phi_{2}^{-}(x) & \text { for } x \leq-\frac{11}{10},
\end{array} \quad \Phi^{+}(x)= \begin{cases}\Phi_{1}^{+}(x) & \text { for } 0 \leq x \leq \frac{6}{5} \\
\Phi_{2}^{+}(x) & \text { for } \frac{6}{5} \leq x<\infty\end{cases}\right.
$$

with

$$
\begin{aligned}
\Phi_{1}^{-}(x)= & -\frac{173007133315}{285311670611}-\frac{67406738275}{69202853326}\left(x+\frac{11}{10}\right) \\
& -\frac{702087707035846036257168105708375}{331414880343242169114421852929976}\left(x+\frac{11}{10}\right)^{2}, \\
\Phi_{2}^{-}(x)= & \frac{1}{4 x}+\frac{1}{4 x^{3}}+\frac{15}{64 x^{5}}+\frac{11}{64 x^{7}}+\frac{19}{512 x^{9}}-\frac{85}{512 x^{11}}, \\
\Phi_{2}^{+}(x)= & -\frac{1}{6 x}-\frac{1}{6 x^{3}}-\frac{35}{216 x^{5}}-\frac{31}{216 x^{7}}-\frac{133}{1296 x^{9}}-\frac{5}{144 x^{11}} \\
& +\frac{15545}{279936 x^{13}}+\frac{41381}{279936 x^{15}}+\frac{1020679}{5038848 x^{17}}+\frac{838775}{5038848 x^{19}},
\end{aligned}
$$

and $\Phi_{1}^{+}(x)$ the polynomial with minimal degree such that $y_{i}=\Phi_{1}^{+}\left(x_{i}\right)$ with

$$
\begin{gathered}
\left(x_{i}, y_{i}\right) \in\left\{\left(0,-\frac{66329}{14331}\right),\left(\frac{6}{35},-\frac{57983}{16009}\right),\left(\frac{12}{35},-\frac{37061}{13765}\right),\left(\frac{18}{35},-\frac{28155}{14903}\right),\right. \\
\left.\left(\frac{24}{35},-\frac{17830}{14261}\right),\left(\frac{6}{7},-\frac{33068}{41763}\right),\left(\frac{36}{35},-\frac{12900}{26023}\right),\left(\frac{6}{5},-\frac{1031522014772972842265}{3070471107232407748608}\right)\right\} .
\end{gathered}
$$

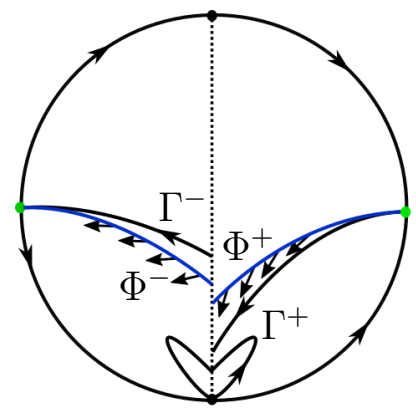

FiguRE 19. Behavior of infinite for $\lambda^{+}=6, \lambda^{-}=-4$ and the blue curves $\Phi^{ \pm}$.

Proof. We prove that the curve $y-\Phi^{+}(x)=0$ (resp. $y-\Phi^{-}(x)=0$ ) is piecewise continuous and without contact with respect to the vector fields $Z^{+}$(resp. $Z^{-}$) when $x \geq 0$ (resp. $x \leq 0$ ). Then, checking some other properties of the functions $\Phi^{ \pm}$, the 
geometrical situations of the points on the curves $\Gamma^{ \pm}$with respect to the graph of $\Phi^{ \pm}(x)$ satisfy the conditions given in the statement. That is, as we have depicted in Figure 19.

The piecewise continuity property follows checking only that $\Phi_{1}^{ \pm}$are polynomials, $\Phi_{2}^{ \pm}$ are rational functions well defined in the interval of definition, and $\Phi_{1}^{+}(6 / 5)=\Phi_{2}^{+}(6 / 5)$ and $\Phi_{1}^{-}(-11 / 10)=\Phi_{2}^{-}(-11 / 10)$. Straightforward computations show that the function $\Phi^{+}(x)$ (resp. $\left.\Phi^{-}(x)\right)$ is monotonous increasing (resp. decreasing). Moreover, for $x \geq 0$,

$$
\left.\left\langle\nabla\left(y-\Phi^{+}(x)\right), Z^{+}\right\rangle\right|_{y=\Phi^{+}(x)}<0
$$

and, for $x \leq 0$,

$$
\left.\left\langle\nabla\left(y-\Phi^{-}(x)\right), Z^{-}\right\rangle\right|_{y=\Phi^{-}(x)}<0 .
$$

These properties follow because all the involved functions, in each considered intervals $\{(-\infty,-11 / 10],[-11 / 10,0],[0,6 / 5],[6 / 5, \infty)\}$, write as $p(x) / x^{k}$, being $p$ polynomials with rational coefficients and $k \geq 0$ integers. Because, $\Phi_{i}^{ \pm}(x)$, for $i=1,2$ are also functions of this type. Moreover, these polynomials $p(x)$ have no zeros in the above intervals.

The proof finishes checking that, when $x$ goes to $+\infty$ (resp. $-\infty), \Gamma^{+} \cap\{x \geq 0\} \subset$ $\left\{y-\Phi^{+}(x)<0\right\}$ (resp. $\Gamma^{-} \cap\{x \leq 0\} \subset\left\{y-\Phi^{-}(x)>0\right\}$ ). This property holds because, when $x \nearrow+\infty$, we have

$$
\left.\left\langle\nabla\left(y-\widehat{\Phi}_{2}^{+}(x)\right), Z^{+}\right\rangle\right|_{y=\widehat{\Phi}_{2}^{+}(x)}>0 \quad \text { and } \quad \widehat{\Phi}_{2}^{+}(x)<\Phi_{2}^{+}(x) .
$$

Equivalently, when $x \searrow-\infty$, we get

$$
\left.\left\langle\nabla\left(y-\widehat{\Phi}_{2}^{-}(x)\right), Z^{-}\right\rangle\right|_{y=\widehat{\Phi}_{2}^{-}(x)}>0 \quad \text { and } \quad \widehat{\Phi}_{2}^{-}(x)>\Phi_{2}^{-}(x) .
$$

Where

$$
\begin{aligned}
& \widehat{\Phi}_{2}^{-}(x)=\Phi_{2}^{-}(x)-\frac{5985}{16384 x^{13}}-\frac{6381}{16384 x^{15}}-\frac{879}{131072 x^{17}}, \\
& \widehat{\Phi}_{2}^{+}(x)=\Phi_{2}^{+}(x)-\frac{838775}{5038848 x^{19}} .
\end{aligned}
$$

Hence, the graph of the curve $\Gamma^{+}$(resp. $\Gamma^{-}$) is between the graphs of the functions $\Phi_{2}^{+}$ and $\widehat{\Phi}_{2}^{+}$(resp. $\Phi_{2}^{-}$and $\widehat{\Phi}_{2}^{-}$) when $x \nearrow+\infty$ (resp. $x \searrow-\infty$ ). See Figure 20.

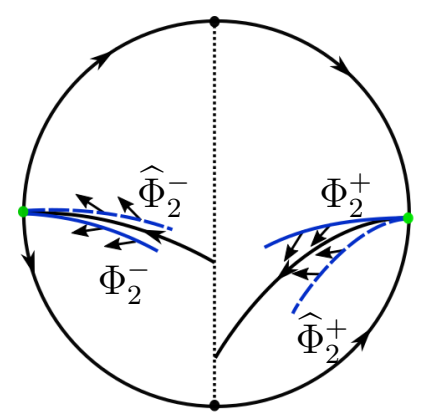

Figure 20. The vector field $Z^{ \pm}$on the without contact curves $y-\Phi_{2}^{ \pm}(x)=$ 0 and $y-\widehat{\Phi}_{2}^{ \pm}(x)=0$.

Next, we prove the existence of a point, in the parameter space, exhibiting the connection at infinity.

Corollary 4.7. There exists $-4<\lambda^{*}<0$ such that system $(6)$ for $\left(\lambda^{+}, \lambda^{-}\right)=\left(6, \lambda^{*}\right)$ exhibits a connection between the semihyperbolic saddles at infinity.

Proof. The proof follows by continuity taking $\lambda^{+}=6$ in Corollary 4.4 and Proposition 4.6. Following the notation introduced in Proposition 4.6, the intersection points with the $x$ axis of the separatrices $\Gamma^{ \pm}$satisfy $\Phi^{+}(0)-\Phi^{-}(0)>0$ for $\lambda^{-} \lesssim 0$ and $\Phi^{+}(0)-\Phi^{-}(0)<0$ for $\lambda^{-}=-4$. See also Figures 17 and 19 . 
Next we explain the procedure to provide the functions $\Phi^{ \pm}$appearing in the previous results. Other previous works where this kind of mechanism is used are $[11,14]$. The function $\Phi^{+}$is a piecewise continuous function defined by $\Phi_{1}^{+}$and $\Phi_{2}^{+}$in two intervals, $\left[0, x_{1}^{+}\right]$and $\left[x_{1}^{+}, \infty\right)$, respectively. Clearly, $\Phi_{1}^{+}\left(x_{1}^{+}\right)=\Phi_{2}^{+}\left(x_{1}^{+}\right)$. The function $\Phi_{2}^{+}$is obtained computing the approximation up to some order $k$ of the unstable separatrix of the saddle located at the origin of system (19), that is, system (6) in the chart $\mathcal{U}_{1}$. Although $\Phi_{2}^{+}$define a curve without contact, it does not cross the $y$-axis. This is why we have considered the two pieces. It is necessary that the function $\Phi_{1}^{+}$maintain the without contact property and such that the graphs arrive to the $y$-axis. For simplicity, we have chosen it as a polynomial of some degree $\ell$. The function $\Phi^{-}$satisfies equivalent conditions. In Proposition 4.6 we have proposed two different ways to determine such polynomials. In both, the initial value problem defined by the original differential system (6) satisfying $y_{1}^{ \pm}=\Phi_{1}^{ \pm}\left(x_{1}^{ \pm}\right)$is considered. The difference is the mechanism to solve them. One is the power series method at $x_{1}^{ \pm}$. The other is the interpolation polynomial defined by the points obtained with the classical Euler method with some step $h$, see [27]. Finally, we need to find adequate values for $k^{ \pm}, \ell^{ \pm}, x_{1}^{ \pm}, h^{ \pm}$, such that the relative positions of the graphs of the functions $\Phi^{ \pm}$allow us to proof a result like Proposition 4.6.

An improvement of the above results is hard to be done because the described mechanism depend on too many parameters and small variations on them have a big effect on all the involved functions. Moreover, the analytic control of the curves $\Gamma^{ \pm}$is very difficult.

Although, the last result provides the existence of a point on the connection curve $\Upsilon$, from the above comments it is clear that the proof of the existence of such bifurcation curve, where the limit cycles disappear, is far to be done. We finish doing some numerical simulations to reinforce the idea that, fixed $\lambda^{+}$, there exists only one value $\lambda^{-}$such that the connection holds. Consequently, the connection curve $\Upsilon$ should be the graph of a function, see Figure 11.

Following the notation introduced in Proposition 4.6, we can define the intersection points $\left(0, \eta^{ \pm}\right)$of the curves $\Gamma^{ \pm}$with the discontinuity line $\Sigma$, which is in this system the $y$-axis. These points define two functions of one variable, $\eta^{ \pm}\left(\lambda^{ \pm}\right)$. By the symmetries of system (6), studied in Lemma 4.1, we have that $\eta^{-}(-\lambda)=\eta^{+}(\lambda)$. Consequently, we compute numerically only $\eta^{+}$. The computations has been made by numerical continuation of the analytic approximations of high order of the invariant manifold $\Gamma^{+}$, denoted by $\Phi_{2}^{+}$ in Proposition 4.6). The graphs of $\eta^{+}$are depicted in Figure 21. The value $\lambda^{+}=\hat{\lambda}$ denotes where the function $\eta^{+}$has its maximum. Notice that $\eta^{+}$is monotonous increasing in $(0, \hat{\lambda})$ and monotonous decreasing in $(\hat{\lambda}, \infty)$.

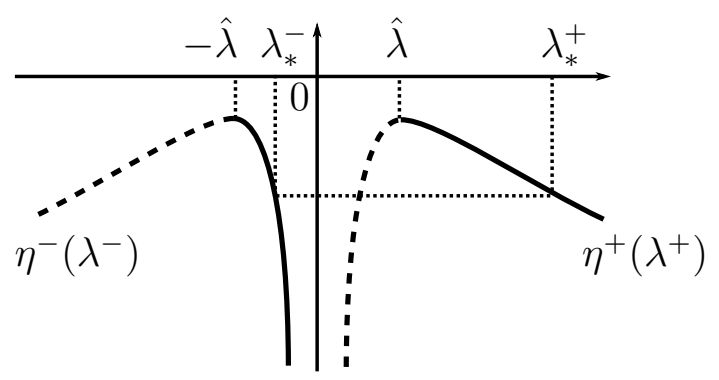

Figure 21. The functions $\eta^{ \pm}$that define the intersection points of $\Gamma^{ \pm}$with $\Sigma$.

The bifurcation curve $\Upsilon$ is defined by the connection of the separatrices $\Gamma^{+}$and $\Gamma^{-}$on the separation curve $\Sigma$. The symmetric value $\hat{\lambda}$, where the functions $\eta^{ \pm}$have a maximum, defines the intersection points of $\Upsilon$ with the bisector, $\lambda^{+}+\lambda^{-}=0$, of the fourth quadrant. 
Using the symmetries indicated in Lemma 4.1 and the plot of $\eta^{ \pm}$in Figure 21, the curve $\Upsilon$ is obtained from the pairs $\left(\lambda_{*}^{+}, \lambda_{*}^{-}\right)$for every negative value less or equal than $\eta^{+}(\hat{\lambda})=$ $\eta^{-}(-\hat{\lambda})$. We have numerical evidences that there is only one pair for $\lambda_{*}^{+} \geq \hat{\lambda}$ and $-\hat{\lambda} \geq$ $\lambda_{*}^{-}<0$. This relation can be seen also in Figure 21 where the pairs associated to points on $\Upsilon$ in Figure 11 are depicted as a continuous line and the symmetric part as a dashed line. All the simulations done here indicates that the limit cycle disappears for the values on such bifurcation curve. Finally, we remark that the curve $\Upsilon$ separates the fourth quadrant in two regions having the infinity and the origin with opposite stability.

\section{ACKNOWLEDGEMENTS}

This work has been realized thanks to the Spanish MINECO grant MTM2016-77278P (FEDER), Catalan AGAUR grant 2017 SGR 1617, and the Brazilian CAPES BEX 13473/13-1.

\section{REFERENCES}

[1] C. Buzzi, C. Pessoa, and J. Torregrosa. Piecewise linear perturbations of a linear center. Discrete Contin. Dyn. Syst., 33(9):3915-3936, 2013.

[2] C. A. Buzzi, T. Carvalho, and R. D. Euzébio. On Poincaré-Bendixson theorem and non-trivial minimal sets in planar nonsmooth vector fields. Publ. Mat., 62(1):113-131, 2018.

[3] C. A. Buzzi, A. Gasull, and J. Torregrosa. Algebraic limit cycles in piecewise linear differential systems. International Journal of Bifurcation and Chaos, 28(03):1850039, 2018.

[4] L. A. Cherkas. The Dulac function for polynomial autonomous systems on a plane. Differ. Uravn., 33(5):689-699, 719, 1997.

[5] C. Chicone. Ordinary differential equations with applications, volume 34 of Texts in Applied Mathematics. Springer, New York, second edition, 2006.

[6] B. Coll, A. Gasull, and R. Prohens. Degenerate Hopf bifurcations in discontinuous planar systems. J. Math. Anal. Appl., 253(2):671-690, 2001.

[7] M. di Bernardo, C. J. Budd, A. R. Champneys, and P. Kowalczyk. Piecewise-smooth dynamical systems, volume 163 of Applied Mathematical Sciences. Springer-Verlag London, Ltd., London, 2008. Theory and applications.

[8] F. Dumortier, J. Llibre, and J. C. Artés. Qualitative theory of planar differential systems. Universitext. Springer-Verlag, Berlin, 2006.

[9] A. F. Filippov. A sufficient condition for the existence of a stable limit cycle for an equation of the second order. Mat. Sbornik N.S., 30(72):171-180, 1952.

[10] A. F. Filippov. Differential equations with discontinuous righthand sides, volume 18 of Mathematics and its Applications (Soviet Series). Kluwer Academic Publishers Group, Dordrecht, 1988.

[11] J. D. García-Saldaña, A. Gasull, and H. Giacomini. Bifurcation values for a family of planar vector fields of degree five. Discrete Contin. Dyn. Syst., 35(2):669-701, 2015.

[12] A. Gasull and H. Giacomini. Upper bounds for the number of limit cycles of some planar polynomial differential systems. Discrete Contin. Dyn. Syst., 27(1):217-229, 2010.

[13] A. Gasull and H. Giacomini. Some applications of the extended Bendixson-Dulac theorem. In Progress and challenges in dynamical systems, volume 54 of Springer Proc. Math. Stat., pages 233252. Springer, Heidelberg, 2013.

[14] A. Gasull, H. Giacomini, and J. Torregrosa. Some results on homoclinic and heteroclinic connections in planar systems. Nonlinearity, 23(12):2977-3001, 2010.

[15] H. Giacomini, J. Llibre, and M. Viano. On the nonexistence, existence and uniqueness of limit cycles. Nonlinearity, 9(2):501-516, 1996.

[16] S.-M. Huan and X.-S. Yang. On the number of limit cycles in general planar piecewise linear systems. Discrete Contin. Dyn. Syst., 32(6):2147-2164, 2012.

[17] Y. Ilyashenko. Centennial history of Hilbert's 16th problem. Bull. Amer. Math. Soc. (N.S.), 39(3):301-354, 2002.

[18] C. Li and J. Llibre. Uniqueness of limit cycles for Liénard differential equations of degree four. $J$. Differential Equations, 252(4):3142-3162, 2012.

[19] S. Li and J. Llibre. Phase portraits of piecewise linear continuous differential systems with two zones separated by a straight line. Preprint, December 2017. 
[20] A. Lins, W. de Melo, and C. C. Pugh. On Liénard's equation. In Geometry and topology (Proc. III Latin Amer. School of Math., Inst. Mat. Pura Aplicada CNPq, Rio de Janeiro, 1976), pages 335-357. Lecture Notes in Math., Vol. 597. Springer, Berlin, 1977.

[21] J. Llibre and E. Ponce. Three nested limit cycles in discontinuous piecewise linear differential systems with two zones. Dyn. Contin. Discrete Impuls. Syst. Ser. B Appl. Algorithms, 19(3):325-335, 2012.

[22] J. Llibre, E. Ponce, and F. Torres. On the existence and uniqueness of limit cycles in Liénard differential equations allowing discontinuities. Nonlinearity, 21(9):2121-2142, 2008.

[23] J. Llibre and A. E. Teruel. Introduction to the qualitative theory of differential systems. Birkhäuser Advanced Texts: Basler Lehrbücher. [Birkhäuser Advanced Texts: Basel Textbooks]. Birkhäuser/Springer, Basel, 2014. Planar, symmetric and continuous piecewise linear systems.

[24] J. C. Medrado and J. Torregrosa. Uniqueness of limit cycles for sewing planar piecewise linear systems. J. Math. Anal. Appl., 431(1):529-544, 2015.

[25] S. L. Shi. A concrete example of the existence of four limit cycles for plane quadratic systems. Sci. Sinica, 23(2):153-158, 1980.

[26] S. Smale. Finding a horseshoe on the beaches of Rio. Math. Intelligencer, 20(1):39-44, 1998.

[27] J. Stoer and R. Bulirsch. Introduction to numerical analysis, volume 12 of Texts in Applied Mathematics. Springer-Verlag, New York, third edition, 2002.

[28] Z. F. Zhang, T. R. Ding, W. Z. Huang, and Z. X. Dong. Qualitative theory of differential equations, volume 101 of Translations of Mathematical Monographs. American Mathematical Society, Providence, RI, 1992.

Departament de Matemàtiques, Universitat Autònoma de Barcelona, 08193 Bellaterra, Barcelona, Catalonia, Spain

Email address: leonardo@mat.uab.cat

Departament de Matemàtiques, Universitat Autònoma de Barcelona, 08193 Bellaterra,

Barcelona, Catalonia, Spain

Email address: torre@mat.uab.cat 